\title{
NORMAL DIRECTION CURVES AND APPLICATIONS
}

\author{
SEZAI KIZILTUĞ, MEHMET ÖNDER, AND YUSUF YAYLI
}

Received 23 December, 2014

\begin{abstract}
In this study, we define a new type of associated curves in the Euclidean 3-space such as normal-direction curve and normal-donor curve. We obtain characterizations for these curves. Moreover, we give applications of normal-direction curves to some special curves such as helix, slant helix, plane curve or normal-direction $(N D)$-normal curves in $E^{3}$. And, we show that slant helices and rectifying curves can be constructed by using normal-direction curves.
\end{abstract}

2010 Mathematics Subject Classification: 53A04

Keywords: associated curve, normal-direction curve, normal-donor curve

\section{INTRODUCTION}

In the curve theory of Euclidean space, the most important subject is to obtain a characterization for a regular curve, since these characterizations allow to classify curves according to some relations. These characterizations can be given for a single curve or for a curve pair. Helix, slant helix, plane curve, spherical curve, etc. are the well-known examples of single special curves $[1,10,12,17,20]$ and these curves, especially the helices, are used in many applications $[2,7,9,16]$. Moreover, special curves can be defined by considering Frenet planes. If the position vector of a space curve always lies on its rectifying, osculating or normal planes, then the curve is called rectifying curve, osculating curve or normal curve, respectively [4]. In the Euclidean space $E^{3}$, rectifying, normal and osculating curves satisfy Cesaro's fixed point condition, i.e., Frenet planes of such curves always contain a particular point $[8,15]$. In particular, there exists a simple relationship between rectifying curves and Darboux vectors (centrodes), which play some important roles in mechanics, kinematics as well as in differential geometry in defining the curves of constant precession [4].

Moreover, special curve pairs are characterized by some relationships between their Frenet vectors or curvatures. Involute-evolute curves, Bertrand curves, Mannheim curves are the well-known examples of curve pairs and studied by some mathematicians $[3,11,14,19,20]$.

Recently, a new curve pair in the Euclidean 3 -space $E^{3}$ has been defined by Choi and Kim [6]. They have considered an integral curve $\gamma$ of a unit vector field $X$ defined 
in the Frenet basis of a Frenet curve $\alpha$ and they have given the definitions and characterizations of principal-directional curve and principal-donor curve in $E^{3}$. They also gave some applications of these curves to some special curves.

In the present paper, we consider a new type of associated curves and define a new curve pair such as normal-direction curve and normal-donor curve in $E^{3}$. We obtain some characterizations for these curves and show that normal-direction curve is a space evolute of normal-donor curve. Moreover, we give some applications of normal-direction curve to some special curves such as helix, slant helix or plane curve.

\section{PRELIMINARIES}

This section includes a brief summary of space curves and definitions of general helix and slant helix in the Euclidean 3-space $E^{3}$.

A unit speed curve $\alpha: I \rightarrow E^{3}$ is called a general helix if there is a constant vector $u$, so that $\langle T, u\rangle=\cos \theta$ is constant along the curve, where $\theta \neq \pi / 2$ and $T(s)=\alpha^{\prime}(s)$ is unit tangent vector of $\alpha$ at $s$. The curvature (or first curvature) of $\alpha$ is defined by $\kappa(s)=\left\|\alpha^{\prime \prime}(s)\right\|$. Then, the curve $\alpha$ is called Frenet curve, if $\kappa(s) \neq 0$, and the unit principal normal vector $N(s)$ of the curve $\alpha$ at $s$ is given by $\alpha^{\prime \prime}(s)=\kappa(s) N(s)$. The unit vector $B(s)=T(s) \times N(s)$ is called the unit binormal vector of $\alpha$ at $s$. Then $\{T, N, B\}$ is called the Frenet frame of $\alpha$. For the derivatives of the Frenet frame, the following Frenet-Serret formulae hold:

$$
\left[\begin{array}{c}
T^{\prime} \\
N^{\prime} \\
B^{\prime}
\end{array}\right]=\left[\begin{array}{ccc}
0 & \kappa & 0 \\
-\kappa & 0 & \tau \\
0 & -\tau & 0
\end{array}\right]\left[\begin{array}{c}
T \\
N \\
B
\end{array}\right]
$$

where $\tau(s)$ is the torsion (or second curvature) of $\alpha$ at $s$. It is well-known that the curve $\alpha$ is a general helix if and only if $\frac{\tau}{\kappa}(s)=$ constant $[17,18]$. If both $\kappa(s) \neq 0$ and $\tau(s)$ are constants, we call $\alpha$ as a circular helix. A curve $\alpha$ with $\kappa(s) \neq 0$ is called a slant helix if the principal normal lines of $\alpha$ make a constant angle with a fixed direction. Also, a slant helix $\alpha$ in $E^{3}$ is characterized by the differential equation of its curvature $\kappa$ and its torsion $\tau$ given by

$$
\frac{\kappa^{2}}{\left(\kappa^{2}+\tau^{2}\right)^{3 / 2}}\left(\frac{\tau}{\kappa}\right)^{\prime}=\text { constant. }
$$

(See [12]).

Now, we give the definitions of some associated curves defined by Choi and Kim [6]. Let $I \subset \mathbb{R}$ be an open interval. For a Frenet curve $\alpha: I \rightarrow E^{3}$, consider a vector field $X$ given by

$$
X(s)=u(s) T(s)+v(s) N(s)+w(s) B(s)
$$


where $u, v$ and $w$ are arbitrary differentiable functions of $s$ which is the arc length parameter of $\alpha$. Let

$$
u^{2}(s)+v^{2}(s)+w^{2}(s)=1,
$$

holds. Then the definitions of $X$-direction curve and $X$-donor curve in $E^{3}$ are given as follows.

Definition 1. (Definition 2.1. in [6]) Let $\alpha$ be a Frenet curve in Euclidean 3-space $E^{3}$ and $X$ be a unit vector field satisfying the equations (2.2) and (2.3). The integral curve $\beta: I \rightarrow E^{3}$ of $X$ is called an $X$-direction curve of $\alpha$. The curve $\alpha$ whose $X$ direction curve is $\beta$ is called the $X$-donor curve of $\beta$ in $E^{3}$.

Definition 2. (Definition 2.2. in [6]) An integral curve of principal normal vector $N(s)$ (resp. binormal vector $B(s)$ ) of $\alpha$ in (2.2) is called the principal-direction curve (resp. binormal-direction curve) of $\alpha$ in $E^{3}$.

Remark 1. (Remark 2.3. in [6]) A principal-direction (resp. the binormal-direction) curve is an integral curve of $X(s)$ with $u(s)=w(s)=0, v(s)=1$ (resp. $u(s)=$ $v(s)=0, w(s)=1)$ for all $s$ in $(2.2)$.

\section{NORMAL-DIRECTION CURVE AND NORMAL-DONOR CURVE IN $E^{3}$}

In this section, we will give definitions of normal-direction curve and normal donor curve in $E^{3}$. We obtain some theorems and results characterizing these curves. First, we give the following definition.

Definition 3. Let $\alpha$ be a Frenet curve in $E^{3}$ and $X$ be a unit vector field lying on the normal plane of $\alpha$ and defined by

$$
X(s)=v(s) N(s)+w(s) B(s), \quad v(s) \neq 0, \quad w(s) \neq 0,
$$

and satisfying that the vectors $X^{\prime}(s)$ and $T(s)$ are linearly dependent. The integral curve $\gamma: I \rightarrow E^{3}$ of $X(s)$ is called a normal-direction curve of $\alpha$. The curve $\alpha$ whose normal -direction curve is $\gamma$ is called the normal-donor curve in $E^{3}$.

The Frenet frame is a rotation-minimizing with respect to the principal normal $N$ [8]. If we consider a new frame given by $\{T, X, M\}$ where $M=T \times X$, we have that this new frame is rotation-minimizing with respect to $T$, i.e., the unit vector $X$ belongs to a rotation-minimizing frame.

Since, $X(s)$ is a unit vector and $\gamma: I \rightarrow E^{3}$ is an integral curve of $X(s)$, without loss of generality we can take $s$ as the arc length parameter of $\gamma$ and we can give the following characterizations in the view of these information.

Theorem 1. Let $\alpha: I \rightarrow E^{3}$ be a Frenet curve and an integral curve of $X(s)=$ $v(s) N(s)+w(s) B(s)$ be the curve $\gamma: I \rightarrow E^{3}$. Then, $\gamma$ is a normal-direction curve of 
$\alpha$ if and only if the following equalities hold,

$$
v(s)=\sin \left(\int \tau d s\right) \neq 0, \quad w(s)=\cos \left(\int \tau d s\right) \neq 0 .
$$

Proof. Since $\gamma$ is a normal-direction curve of $\alpha$, from Definition 3, we have

$$
X(s)=v(s) N(s)+w(s) B(s),
$$

and

$$
v^{2}(s)+w^{2}(s)=1 .
$$

Differentiating (3.3) with respect to $s$ and by using the Frenet formulas, it follows

$$
X^{\prime}(s)=-v \kappa T+\left(v^{\prime}-w \tau\right) N+\left(w^{\prime}+v \tau\right) B .
$$

Since we have that $X^{\prime}$ and $T$ are linearly dependent. Then from (3.5) we can write

$$
\left\{\begin{array}{l}
-v \kappa \neq 0 \\
v^{\prime}-w \tau=0 \\
w^{\prime}+v \tau=0
\end{array}\right.
$$

The solutions of second and third differential equations are

$$
v(s)=\sin \left(\int \tau d s\right) \neq 0, \quad w(s)=\cos \left(\int \tau d s\right) \neq 0,
$$

respectively, which completes the proof.

Theorem 2. Let $\alpha: I \rightarrow E^{3}$ be a Frenet curve. If $\gamma$ is the normal-direction curve of $\alpha$, then $\gamma$ is a space evolute of $\alpha$.

Proof. Since $\gamma$ is an integral curve of $X$, we have $\gamma^{\prime}=X$. Denote the Frenet frame of $\gamma$ by $\{\bar{T}, \bar{N}, \bar{B}\}$. Differentiating $\gamma^{\prime}=X$ with respect to $s$ and by using Frenet formulas we get

$$
X^{\prime}=\bar{T}^{\prime}=\bar{\kappa} \bar{N} .
$$

Furthermore, we know that $X^{\prime}$ and $T$ are linearly dependent. Then from (3.7) we get $\bar{N}$ and $T$ are linearly dependent, i.e, $\gamma$ is a space evolute of $\alpha$.

Theorem 3. Let $\alpha: I \rightarrow E^{3}$ be a Frenet curve. If $\gamma$ is the normal direction curve of $\alpha$, then the curvature $\bar{\kappa}$ and the torsion $\bar{\tau}$ of $\gamma$ are given as follows,

$$
\bar{\kappa}=\kappa\left|\sin \left(\int \tau d s\right)\right|, \quad \bar{\tau}=\kappa \cos \left(\int \tau d s\right) .
$$

Proof. From (3.5), (3.6) and (3.7), we have

$$
\bar{\kappa} \bar{N}=-v \kappa T \text {. }
$$

By considering (3.8) and (3.2) we obtain

$$
\bar{\kappa} \bar{N}=-\kappa \sin \left(\int \tau d s\right) T
$$


which gives us

$$
\bar{\kappa}=\kappa\left|\sin \left(\int \tau d s\right)\right|
$$

Moreover, from (3.9) and (3.10), we can write

$$
\bar{N}=T \text {. }
$$

Then, we have

$$
\bar{B}=\bar{T} \times \bar{N}=\cos \left(\int \tau d s\right) N-\sin \left(\int \tau d s\right) B .
$$

Differentiating (3.12) with respect to $s$ gives

$$
\bar{B}^{\prime}=-\kappa \cos \left(\int \tau d s\right) T .
$$

Since $\bar{\tau}=-\left\langle\bar{B}^{\prime}, \bar{N}\right\rangle=-\left\langle\bar{B}^{\prime}, T\right\rangle$, from (3.13) it follows

$$
\bar{\tau}=\kappa \cos \left(\int \tau d s\right)
$$

that finishes the proof.

Corollary 1. Let $\gamma$ be a normal-direction curve of the curve $\alpha$. Then the relationships between the Frenet frames of curves are given as follows,

$$
\begin{aligned}
& X=\bar{T}=\sin \left(\int \tau d s\right) N+\cos \left(\int \tau d s\right) B, \\
& \bar{N}=T, \\
& \bar{B}=\cos \left(\int \tau d s\right) N-\sin \left(\int \tau d s\right) B .
\end{aligned}
$$

Proof. The proof is clear from Theorem 3.

Theorem 4. Let $\gamma$ be a normal-direction curve of $\alpha$ with curvature $\overline{\mathrm{K}}$ and torsion $\bar{\tau}$. Then curvature $\kappa$ and torsion $\tau$ of $\alpha$ are given by

$$
\kappa=\sqrt{\bar{\kappa}^{2}+\bar{\tau}^{2}}, \quad \tau=\frac{\bar{\tau}^{2}}{\bar{\kappa}^{2}+\bar{\tau}^{2}}\left(\frac{\bar{\kappa}}{\bar{\tau}}\right)^{\prime} .
$$

Proof. From (3.10) and (3.14), we easily get

$$
\kappa=\sqrt{\bar{\kappa}^{2}+\bar{\tau}^{2}} .
$$

Substituting (3.15) into (3.10) and (3.14), it follows

$$
\left|\sin \left(\int \tau d s\right)\right|=\frac{\bar{\kappa}}{\sqrt{\bar{\kappa}^{2}+\bar{\tau}^{2}}},
$$




$$
\cos \left(\int \tau d s\right)=\frac{\bar{\tau}}{\sqrt{\bar{\kappa}^{2}+\bar{\tau}^{2}}},
$$

respectively. Differentiating (3.16) with respect to $s$, we have

$$
\tau \cos \left(\int \tau d s\right)=\frac{\bar{\tau}\left(\bar{\kappa}^{\prime} \bar{\tau}-\bar{\kappa} \bar{\tau}^{\prime}\right)}{\left(\bar{\kappa}^{2}+\bar{\tau}^{2}\right)^{3 / 2}} .
$$

From (3.17) and (3.18), it follows

$$
\tau=\frac{\bar{\kappa}^{\prime} \bar{\tau}-\bar{\kappa} \bar{\tau}^{\prime}}{\bar{\kappa}^{2}+\bar{\tau}^{2}},
$$

or equivalently,

$$
\tau=\frac{\bar{\tau}^{2}}{\bar{\kappa}^{2}+\bar{\tau}^{2}}\left(\frac{\bar{\kappa}}{\bar{\tau}}\right)^{\prime}
$$

Theorem 4 leads us to give the following corollary whose proof is clear.

Corollary 2. Let $\gamma$ with the curvature $\bar{\kappa}$ and the torsion $\bar{\tau}$ be a normal-direction curve of $\alpha$. Then

$$
\frac{\tau}{\kappa}=-\frac{\bar{\kappa}^{2}}{\left(\bar{\kappa}^{2}+\bar{\tau}^{2}\right)^{3 / 2}}\left(\frac{\bar{\tau}}{\bar{\kappa}}\right)^{\prime},
$$

is satisfied, where $\kappa$ and $\tau$ are curvature and torsion of $\alpha$, respectively.

\section{APPLICATIONS OF NORMAL-DIRECTION CURVES}

In this section, we focus on relations between normal-direction curves and some special curves such as general helix, slant helix, plane curve or rectifying curve in $E^{3}$.

4.1. General helices, slant helices and plane curves

Considering Corollary 2, we have the following theorems which gives a way to construct the examples of slant helices by using general helices.

Theorem 5. Let $\alpha: I \rightarrow E^{3}$ be a Frenet curve in $E^{3}$ and $\gamma$ be a normal-direction curve of $\alpha$. Then the followings are equivalent,

(i) A Frenet curve $\alpha$ is a general helix in $E^{3}$.

(ii) $\alpha$ is a normal-donor curve of a slant helix.

(iii) A normal-direction curve of $\alpha$ is a slant helix.

Theorem 6. Let $\alpha: I \rightarrow E^{3}$ be a Frenet curve in $E^{3}$ and $\gamma$ be a normal-direction curve of $\alpha$. Then the followings are equivalent,

(i) A Frenet curve $\alpha$ is a plane curve in $E^{3}$.

(ii) $\alpha$ is a normal-donor curve of a general helix. 
(iii) A normal-direction curve of $\alpha$ is a general helix.

Example 1. Let consider the general helix given by the parametrization $\alpha(s)=\left(\cos \frac{s}{\sqrt{2}}, \sin \frac{s}{\sqrt{2}}, \frac{s}{\sqrt{2}}\right)$ in $E^{3}$ (Fig 1a). The Frenet vectors and curvatures of $\alpha$ are obtained as follows,

$$
\begin{aligned}
T(s) & =\left(-\frac{1}{\sqrt{2}} \sin \frac{s}{\sqrt{2}}, \frac{1}{\sqrt{2}} \cos \frac{s}{\sqrt{2}}, \frac{1}{\sqrt{2}}\right), \\
N(s) & =\left(-\cos \frac{s}{\sqrt{2}}, \sin \frac{s}{\sqrt{2}}, 0\right), \\
B(s) & =\left(\frac{1}{\sqrt{2}} \sin \frac{s}{\sqrt{2}},-\frac{1}{\sqrt{2}} \cos \frac{s}{\sqrt{2}}, \frac{1}{\sqrt{2}}\right), \\
\kappa & =\tau=\frac{1}{2} .
\end{aligned}
$$

Then we have $X(s)=\left(x_{1}(s), x_{2}(s), x_{3}(s)\right)$ where

$$
\begin{aligned}
& x_{1}(s)=-\sin \left(\frac{s}{2}+c\right) \cos \frac{s}{\sqrt{2}}+\frac{1}{\sqrt{2}} \cos \left(\frac{s}{2}+c\right) \sin \frac{s}{\sqrt{2}}, \\
& x_{2}(s)=\sin \left(\frac{s}{2}+c\right) \sin \frac{s}{\sqrt{2}}-\frac{1}{\sqrt{2}} \cos \left(\frac{s}{2}+c\right) \cos \frac{s}{\sqrt{2}}, \\
& x_{3}(s)=\frac{1}{\sqrt{2}} \cos \left(\frac{s}{2}+c\right) .
\end{aligned}
$$

and $c$ is integration constant. Now, we can construct a slant helix $\gamma$ which is also a normal-direction curve of $\alpha$ (Fig 1b):

$$
\gamma=\int_{0}^{s} \gamma^{\prime}(s) d s=\int_{0}^{s} X(s) d s=\left(\gamma_{1}(s), \gamma_{2}(s), \gamma_{3}(s)\right)
$$

where

$$
\begin{aligned}
& \gamma_{1}(s)=\int_{0}^{s}\left[-\sin \left(\frac{s}{2}+c\right) \cos \frac{s}{\sqrt{2}}+\frac{1}{\sqrt{2}} \cos \left(\frac{s}{2}+c\right) \sin \frac{s}{\sqrt{2}}\right] d s, \\
& \gamma_{2}(s)=\int_{0}^{s}\left[\sin \left(\frac{s}{2}+c\right) \sin \frac{s}{\sqrt{2}}-\frac{1}{\sqrt{2}} \cos \left(\frac{s}{2}+c\right) \cos \frac{s}{\sqrt{2}}\right] d s, \\
& \gamma_{3}(s)=\int_{0}^{s} \frac{1}{\sqrt{2}} \cos \left(\frac{s}{2}+c\right) d s .
\end{aligned}
$$




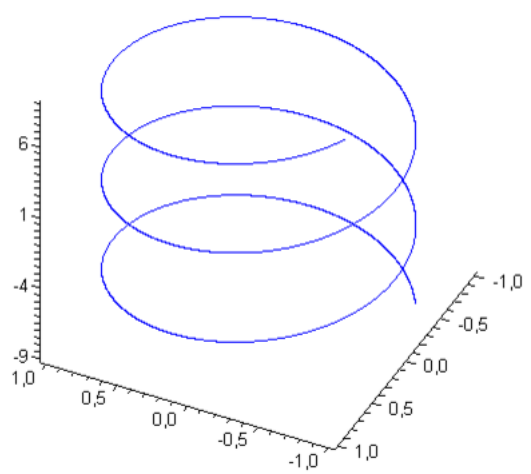

(A) General helix $\alpha$.

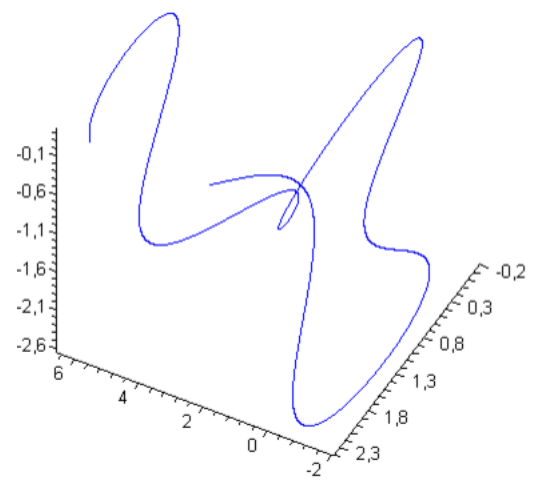

(B) Slant helix $\gamma$.

FIGURE 1. Slant helix $\gamma$ constructed by $\alpha$.

\subsection{ND-normal Curves}

In this subsection we define normal-direction $(N D)$-normal curves in $E^{3}$ and give the relationships between normal-direction curves and $N D$-normal curves.

A space curve whose position vector always lies in its normal plane is called normal curve [5]. Moreover, if the Frenet frame and curvatures of a space curve are given by $\{T, N, B\}$ and $\kappa, \tau$, respectively, then the vector $\tilde{D}(s)=\frac{\tau}{\kappa}(s) T(s)+B(s)$ is called modified Darboux vector of the curve $[12,13]$.

Let now $\alpha$ be a Frenet curve with Frenet frame $\{T, N, B\}$ and $\gamma$ a normal-direction curve of $\alpha$. The curve $\gamma$ is called normal-direction normal curve (or $N D$-normal curve) of $\alpha$, if the position vector of $\gamma$ always lies on the normal plane of its normaldonor curve $\alpha$.

The definition of $N D$-normal curve allows us to write the following equality,

$$
\gamma(s)=m(s) N(s)+n(s) B(s),
$$

where $m(s), n(s)$ are non-zero differentiable functions of $s$. Since $\gamma$ is normaldirection curve of $\alpha$, from Corollary 1 , we have

$$
\left\{\begin{array}{l}
N=\sin \left(\int \tau d s\right) \bar{T}+\cos \left(\int \tau d s\right) \bar{B} \\
B=\cos \left(\int \tau d s\right) \bar{T}-\sin \left(\int \tau d s\right) \bar{B}
\end{array}\right.
$$

Substituting (4.2) in (4.1) gives

$$
\begin{aligned}
\gamma(s)=\left[m \sin \left(\int \tau d s\right)+n \cos \left(\int \tau d s\right)\right] \bar{T} \\
+\left[m \cos \left(\int \tau d s\right)-n \sin \left(\int \tau d s\right)\right] \bar{B}
\end{aligned}
$$


Writing

$$
\left\{\begin{array}{l}
\rho(s)=m \sin \left(\int \tau d s\right)+n \cos \left(\int \tau d s\right) \\
\sigma(s)=m \cos \left(\int \tau d s\right)-n \sin \left(\int \tau d s\right)
\end{array}\right.
$$

in (4.3) and differentiating the obtained equality we obtain

$$
\bar{T}=\rho^{\prime} \bar{T}+(\rho \bar{\kappa}-\sigma \bar{\tau}) \bar{N}+\sigma^{\prime} \bar{B} .
$$

Then we have

$$
\sigma=a=\text { constant }, \quad \rho=s+b=\frac{\bar{\tau}}{\bar{\kappa}} a,
$$

where $a, b$ are non-zero integration constants. From (4.6), it follows that

$$
\gamma(s)=a\left(\frac{\bar{\tau}}{\bar{\kappa}} \bar{T}+\bar{B}\right)(s)=a \tilde{\bar{D}}(s),
$$

where $\tilde{\bar{D}}$ is the modified Darboux vector of $\gamma$.

Now we can give the followings which characterize $N D$-normal curves.

Theorem 7. Let $\alpha: I \rightarrow E^{3}$ be a Frenet curve in $E^{3}$ and $\gamma$ be a normal-direction curve of $\alpha$. If $\gamma$ is a ND-normal curve in $E^{3}$, then we have the followings,

(i) $\gamma$ is a rectifying curve in $E^{3}$ whose curvatures satisfy $\overline{\bar{\tau}}=\frac{s+b}{a}$ where $a, b$ are non-zero constants .

(ii) The position vector and modified Darboux vector $\tilde{\bar{D}}$ of $\gamma$ are linearly dependent.

Theorem 7 gives a way to construct a rectifying curve by using normal-donor curve as follows:

Corollary 3. Let $\alpha: I \rightarrow E^{3}$ be a Frenet curve in $E^{3}$ and $\gamma$ a ND-normal curve of $\alpha$ in $E^{3}$. Then the position vector of $\gamma$ is obtained as follows,

$$
\begin{aligned}
\gamma(s)= & {\left[(s+b) \sin \left(\int \tau d s\right)+a \cos \left(\int \tau d s\right)\right] N(s) } \\
& +\left[(s+b) \cos \left(\int \tau d s\right)-a \sin \left(\int \tau d s\right)\right] B(s)
\end{aligned}
$$

where $a, b$ are non-zero integration constants.

Proof. The proof is clear from (4.1), (4.4) and (4.6).

Example 2. Let consider the general helix given by the parametrization

and drawn in Fig 2 a.

$$
\alpha(s)=\left(\sqrt{1+s^{2}}, s, \ln \left(s+\sqrt{1+s^{2}}\right)\right),
$$




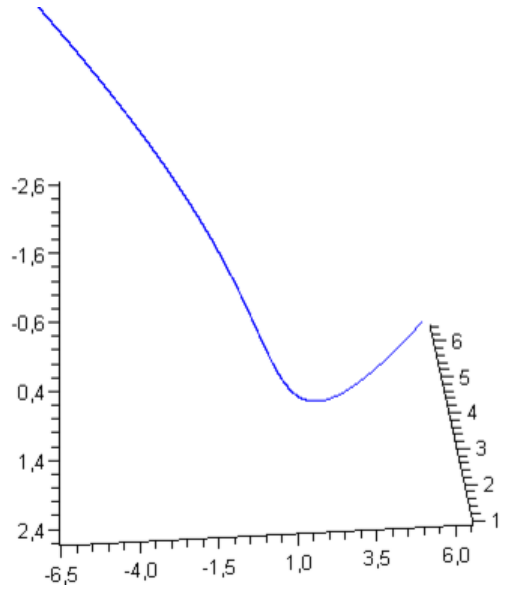

(A) General helix $\alpha$.

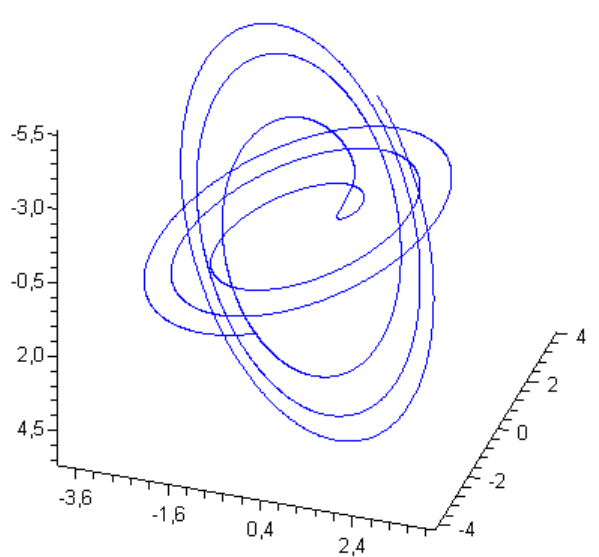

(C) $N D$-normal curve $\gamma$ for $\frac{-3 \pi}{2} \leq s \leq \frac{3 \pi}{2}$.

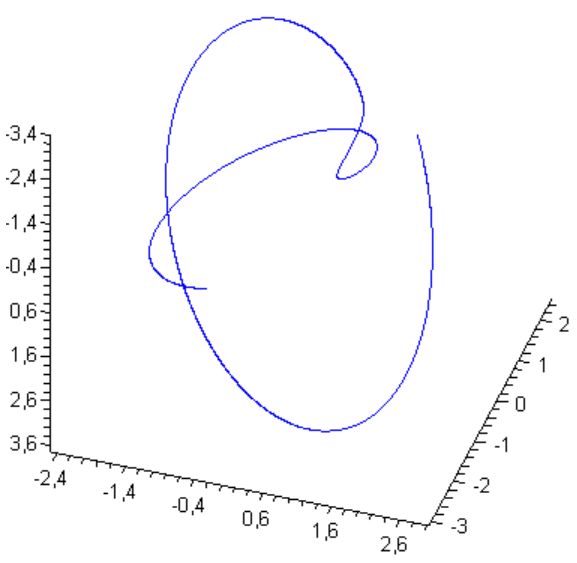

(в) $N D$-normal curve $\gamma$ for $-\pi \leq s \leq \pi$.

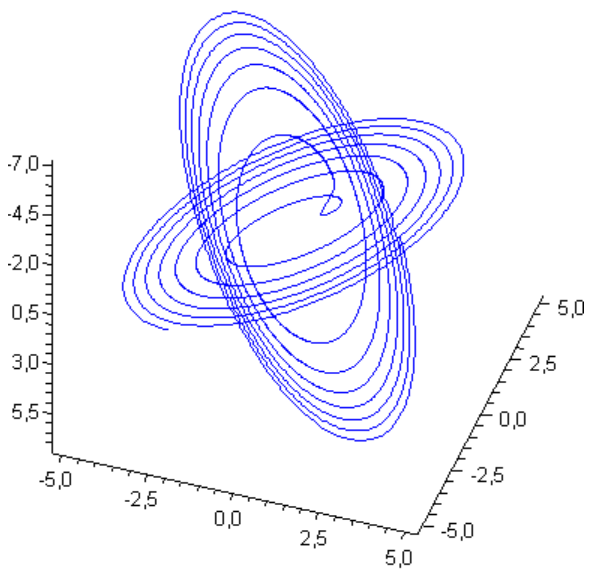

(D) $N D$-normal curve $\gamma$ for $-2 \pi \leq s \leq 2 \pi$.

FIGURE 2. $N D$-normal curve $\gamma$ constructed by $\alpha$.

Frenet vectors and curvatures of the curve are

$$
\begin{aligned}
T(s) & =\frac{1}{\sqrt{2} \sqrt{1+s^{2}}}\left(s, \sqrt{1+s^{2}}, 1\right), \\
N(s) & =\frac{1}{\sqrt{1+s^{2}}}(1,0,-s), \\
B(s) & =\frac{1}{\sqrt{2} \sqrt{1+s^{2}}}\left(-s, \sqrt{1+s^{2}},-1\right), \\
\kappa & =\tau=\frac{1+s^{2}}{2}
\end{aligned}
$$


respectively. Then from Corollary 3 , a $N D$-normal curve $\gamma$ is obtained as follows,

$$
\begin{aligned}
\gamma(s)= & \left(\frac{1}{\sqrt{1+s^{2}}}\left[(s+b) \sin \left(\frac{s}{2}+\frac{s^{3}}{6}+c\right)+a \cos \left(\frac{s}{2}+\frac{s^{3}}{6}+c\right)\right]\right. \\
& -\frac{s}{\sqrt{2\left(1+s^{2}\right)}}\left[(s+b) \cos \left(\frac{s}{2}+\frac{s^{3}}{6}+c\right)-a \sin \left(\frac{s}{2}+\frac{s^{3}}{6}+c\right)\right], \\
& -\frac{1}{\sqrt{2}}\left[(s+b) \cos \left(\frac{s}{2}+\frac{s^{3}}{6}+c\right)-a \sin \left(\frac{s}{2}+\frac{s^{3}}{6}+c\right)\right], \\
& -\frac{s}{\sqrt{1+s^{2}}}\left[(s+b) \sin \left(\frac{s}{2}+\frac{s^{3}}{6}+c\right)+a \cos \left(\frac{s}{2}+\frac{s^{3}}{6}+c\right)\right] \\
& \left.-\frac{1}{\sqrt{2\left(1+s^{2}\right)}}\left[(s+b) \cos \left(\frac{s}{2}+\frac{s^{3}}{6}+c\right)-a \sin \left(\frac{s}{2}+\frac{s^{3}}{6}+c\right)\right]\right)
\end{aligned}
$$

which is also a rectifying curve in the view of Theroem 7 and drawn in Figures $2 b$, $2 \mathrm{c}, 2 \mathrm{~d}$ by choosing $a=b=1, c=0$.

\section{ACKNOWLEDGEMENT}

The authors express their gratitude to the editor and referees for their constructive criticism and feedback, which helped to develop the article.

\section{REFERENCES}

[1] M. Barros, "General helices and a theorem of Lancret." Proc. Amer. Math. Soc., vol. 125, no. 5, pp. 1503-1509, 1997, doi: 10.1090/S0002-9939-97-03692-7.

[2] J. Beltran and J. Monterde, "A characterization of quintic helices." J. Comput. Appl. Math., vol. 206, no. 1, pp. 116-121, 2007, doi: 10.1016/j.cam.2006.06.001.

[3] J. Burke, "Bertrand curves associated with a pair of curves." Mathematics Magazine., vol. 34, no. 1, pp. 60-62, 1960, doi: 10.1080/0025570X.1960.11975181.

[4] B. Y. Chen, "When does the position vector of a space curve always lie in its normal plane?" Amer. Math. Monthly, vol. 110, pp. 147-152, 2003, doi: 10.2307/3647775.

[5] B. Y. Chen and F. Dillen, "Rectifying curves as centrodes and extremal curves." Bull. Inst. Math. Academia Sinica., vol. 33, no. 2, pp. 77-90, 2005.

[6] J. H. Choi and Y. H. Kim, "Associated curves of a Frenet curve and their applications," Applied Mathematics and Computation, vol. 218, no. 18, pp. 9116-9124, 2012, doi: 10.1016/j.amc.2012.02.064.

[7] A. F. Da Fonseca and C. P. Malta, "Lancret helices," arXiv:physics/0507105v1. [Online]. Available: https://arxiv.org/pdf/physics/0507105.pdf

[8] R. Farouki, G. Gianelli, M. Sampoli, and A. Sestini, "Rotation-minimizing osculating frames." Computer Aided Geometric Design., vol. 31, no. 1, pp. 27-42, 2014, doi: 10.1016/j.cagd.2013.11.003.

[9] R. Farouki, C. Y. Han, C. Manni, and A. Sestini, "Characterization and construction of helical polynomial space curves." J. Comput. Appl. Math., vol. 162, no. 2, pp. 365-392, 2004, doi: 10.1016/j.cam.2003.08.030.

[10] H. A. Hayden, "On a generalized helix in a Riemannian n-space." Proc. Lond. Math. Soc., vol. 32, pp. 337-345, 1931, doi: 10.1112/plms/s2-32.1.337. 
[11] S. Izumiya and N. Takeuchi, "Generic properties of helices and Bertrand curves." Journal of Geometry., vol. 74, pp. 97-109, 2002, doi: 10.1007/PL00012543.

[12] S. Izumiya and N. Takeuchi, "New special curves and developable surfaces." Turk. J. Math., vol. 28, pp. 153-163, 2004.

[13] T. Kahraman, M. Önder, M. Kazaz, and H. H. Uğurlu, "Some Characterizations of Mannheim Partner Curves in Minkowski 3-space." Proceedings of the Estonian Academy of Sciences., vol. 60, no. 4, pp. 210-220, 2011, doi: 10.3176/proc.2011.4.02.

[14] H. Liu and F. Wang, "Mannheim partner curves in 3-space." Journal of Geometry., vol. 88, no. 1-2, pp. 120-126, 2008, doi: 10.1007/s00022-007-1949-0.

[15] T. Otsuki, Differential Geometry. Tokyo: Asakura Publishing Co.Ltd., 1961.

[16] J. Puig-Pey, A. Gálvez, and A. Iglesias, "Helical curves on surfaces for computer-aided geometric design and manufacturing." Computational Science and its Applications ICCSA., vol. 3044, no. 2, pp. 771-778, 2004, doi: 10.1007/978-3-540-24709-8_81.

[17] D. J. Struik, Lectures on Classical Differential Geometry., ser. 2. Dover: Addison Wesley., 1988.

[18] F. Wang and H. Liu, "Mannheim partner curves in 3-Euclidean space." Mathematics in Practice and Theory., vol. 37, no. 1, pp. 141-143, 2007.

[19] J. K. Whittemore, "Bertrand curves and helices.” Duke Math. J., vol. 6, no. 1, pp. 235-245, 1950.

[20] Y. C. Wong, "On the generalized helices of Hayden and Syptak in an $N$-space." Proc. Cambridge Philos. Soc., vol. 37, pp. 229-243, 1941, doi: 10.1017/S0305004100021721.

Authors' addresses

Sezai Kızıltuğ

Erzincan Binali Yıldırım University, Faculty of Arts and Sciences, Department of Mathematics, 24030, Erzincan, Turkey

E-mail address: skiziltugeerzincan.edu.tr

Mehmet Önder

Delibekirli Village, Tepe Street, 31440, Kırıkhan, Hatay, Turkey

E-mail address: mehmetonder197999@gmail.com

\section{Yusuf Yaylı}

Ankara University, Faculty of Sciences, Department of Mathematics, 06100, Ankara, Turkey

E-mail address: yayli@science.ankara.edu.tr 